\title{
Performance Evaluation of RPL Objective Function: A Case of Contiki Operation System
}

\author{
Innocent Uzougbo Onwuegbuzie ${ }^{1 *}$, Ajibade Samuel-Soma², Fele Taiwo ${ }^{3}$, Akinwamide \\ Sunday ${ }^{4}$ \\ Department of Computer Science, The Federal Polytechnic Ado-Ekiti, Ekiti State, Nigeria. \\ ${ }^{1}$ onwuegbuzie_iu@fedpolyado.edu.ng, 2 ajibade_ms@fedpolyado.edu.ng, ${ }_{3}^{3}$ fele_ta@fedpolyado.edu.ng, \\ 4akinwamide_so@fedpolyado.edu.ng
}

\begin{abstract}
Wireless Sensor Networks (WSN) is the network of the resource-constrained network which forms the foundation of the Internet of Things (IoT). The Routing Protocol for Low-power and Lossy Networks (RPL) is responsible for generating and managing data routing paths. Nodes implementing RPL uses the mechanics of Objective Function (OF) to select the preferred next-hop node - parent node, and optimal routing path to the destination node. If routing decisions are not efficiently made, this results in increased collision domain, leading to packet losses and packet retransmission which impairs the network operational lifetime. In this study, we present the Contiki Operating System (OS), a state-of-the-art OS for IoTs, ContikiRPL; Contiki variant of RPL. We investigated the performance of RPL with respect to its two OFs; Objective Function Zero (OF0) and the Minimum Rank with Hysteresis Objective Function (MRHOF). The performance of these OFs was evaluated on the following metrics; Packet Delivery Ratio (PDR), Power consumption, and network latency. The result shows that MRHOF outperformed OF0 on all metrics with an overall average PDR of $91.5 \%$, a latency of $44 \mathrm{~ms}$, and power consumption of $1.72 \mathrm{~mW}$ across all nodes. This results in optimal network performance with improved network operational lifetime.
\end{abstract}

Keywords: RPL; Contiki Operating System; Objective Function; Wireless Sensor Networks; Routing Metrics.

*Corresponding author. Tel.: +2347031182647.

E-mail address: onwuegbuzie_in@fedpolyado.edu.ng. 


\section{Introduction}

A wireless sensor network (WSN) is a network of resource-constrained devices that forms the network also known as Low power and lossy network (LLN) [1]. As the bedrock of the Internet of Things (IoT), the devices that form this network are called sensor nodes. Compared with the standard computer system, a unit of a sensor node is constrained by power (mostly battery), memory in the form of Read-only memory (ROM) and Random-Access Memory (RAM), Microcontroller. Other components that make up the sensor node are made of are sensor, a wireless transceiver (radio) - which senses and converts sensed analogue signal to a digital signal using the Analogue to Digital Converter (ADC) [2]. Figure 1 shows the architecture of WSN

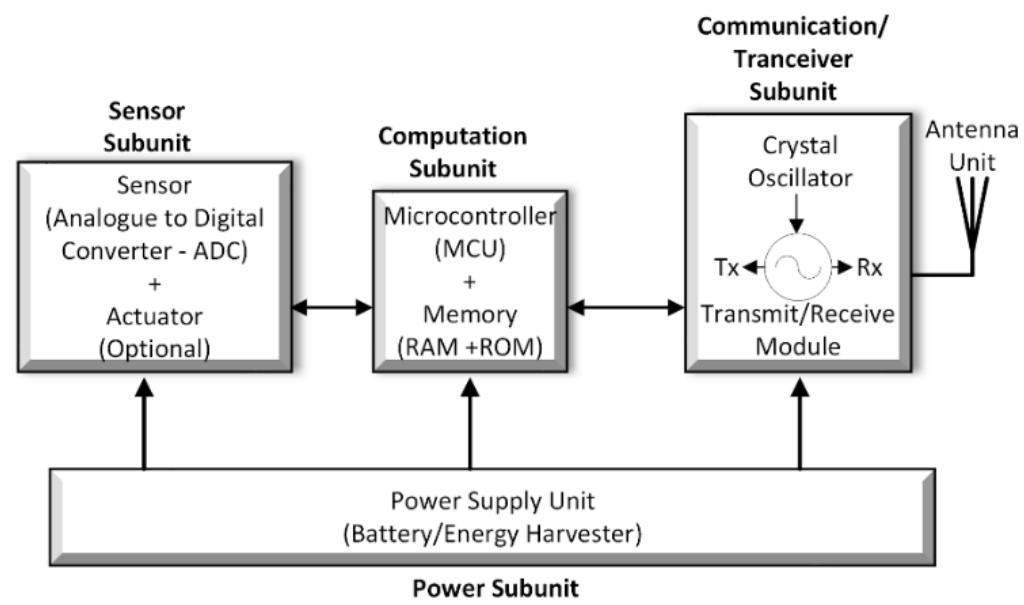

Fig. 1: Architecture of Wireless Sensor Node

WSN is practically used to monitor, sense and report sensed parameters to locations that may be within or remote to the monitoring field. Examples of monitored parameters are; temperature, humidity, pressure, motion, and voltage [3].

The wireless transceiver module transmits and receives radio signals. The miniaturized form factor of the sensor node has mainly resulted in its resource-constrained nature as all of its operational components are built small in size. This design is intentional as it makes it less expensive, easy to replace, and easy to 
install, in locations that may require little or no maintenance. It is mostly powered by either battery, energy scavenging like solar cells, or mains powered [4]. Operating in the $2.4 \mathrm{GHz}$ free license band [5], the traffic generated by WSN is usually termed lossy, due to its dynamically changing nature, making it prone to external interferences. LLNs are aimed for low traffic applications. Low traffic is very common for smart home applications, ubiquitous computing where a very long life is required.

WSN supports three traffic types: Point-to-Point - P2P (between devices inside the LLN), Point-toMultipoint - P2MP (between the central device and other devices inside LLN), and Multipoint-to-Point MP2P (between the devices inside the LLN to a central device) [6]. See Fig 1. A routing protocol or mechanism is used to transport data from one point to the other in the network. It is responsible for creating, managing the routes among sensor nodes, and forwarding data packets through the most optimal path between the source and destination nodes. The lossy nature of the WSN wireless medium has a strong impact on the routing protocol design. Been mindful of this, the routing mechanism must be robust enough to accommodate link lossiness and not react too spontaneously to every changing link as this may lead to the excessive power consumption of the already constrained nodes. Due to these reasons, one of the challenging issues in wireless sensor networks is finding the best routes for the delivery of data, which implies a very efficient routing mechanism for finding and keeping the routes in the network. 


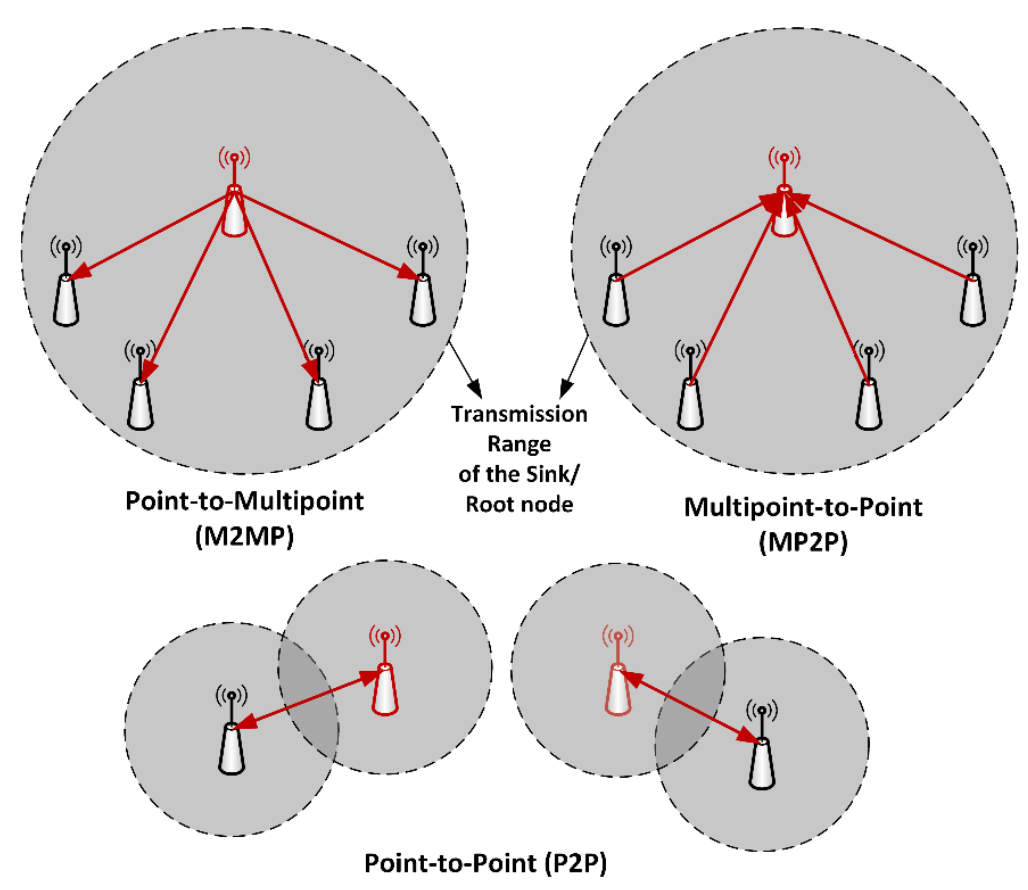

Fig. 2: WSN Communication Patterns

\section{Literature Review}

Routing Protocol for Low-power And Lossy Networks (RPL) [7] is the standard routing protocol presented by the Internet Engineering Task Force (IETF) [8]. It is an IPv6 compliant protocol for low power and lossy networks designed, by the Routing Over Low-power and Lossy network (ROLL) group of IETF [9]. RPL is a distance-vector proactive routing protocol which starts to find routes as soon as the network is powered on. The protocol forms a tree-like topology known as Destination Oriented Directed Acyclic Graph (DODAG) [10]. The DODAG is a virtual routing graph of interconnected formation of child and parent nodes through a multihop pattern linking the source to the destination node. Child nodes forward data to preferred parent nodes until the data arrives at the destination nodes. All node has a routing table that stores the lists of all neigbouring nodes, the size of the routing table increases as one move towards the root node. Path selection is an intelligent decision that is made by considering some factors called metrics and constraints such as Objective Functions (OF) and routing metrics. 
By the standard, RPL uses two major objective functions to generate routing paths between the source and destination nodes. These are, Objective Function Zero (OF0) [10] and Minimum Rank with Hysteresis Objective Function (MRHOF) [11]. OF0 uses the Hop Count (HC) metric to establish a routing path leading to the root node from the source node. Amongst multiple paths, the path with the minimum or least $\mathrm{HC}$ is selected as the preferred routing path by a node. On the other hand, MRHOF adopts other stringent measures to establish a possible routing path. MRHOF uses either a single or the combination of the following metrics to generate routing paths; Expected Transmission Cost (ETX) [8] - which define how many repeated transmissions was undertaken before a packet arrived at the destination node, Latency - which is End-to-End (E2E) packet delay, node Residual Energy - which is the instantaneous energy of a node before it is completely drained out. MRHOF additively or multiplicatively uses either or the combination of these metrics to generate a routing path and the least or minimum path cost is selected as the preferred path.

The lossy nature of WSN does not support predefined steady network topology or routing paths/graphs, as the LLN routing paths are dynamically changing due to constantly changing link qualities. Data are routed in the upwards direction from child nodes to parent nodes all the way leading to the destination or root node, while control messages used to build and maintain the routing paths are propagated in both upwards and downwards directions.

RPL uses the Internet Control Messaging Protocol (ICMP) control messages to build and maintain routing paths [12]. The control messages are DODAG Information Object (DIO), DODAG Information Solicitation (DIS), DODAG Destination Advertisement Object (DAO), DODAG Destination Advertisement Object with Acknowledgement (DAO-ACK) [13]. DIO messages are used by RPL to form, maintain, and discover the DODAG. When an RPL network starts, the nodes start exchanging the information about the DODAG using DIO messages which contain information about the DODAG configuration and help the nodes to join the DODAG and select parents. The DIS is used by any node to explicitly solicit the DIO messages from the neighbour nodes. It is triggered by the node in a case when it 
could not receive a DIO after a predefined time interval. The DAO messages are used by RPL to propagate a node prefix to the ancestor nodes in support of downward traffic.

The lossy wireless links and resource-constrained nature of WSN, coupled with the various routing requirements and high flexibility of RPL configuration poses a challenge to proposing an optimal routing mechanism. Over the years, researchers have actively proposed improvements to the RPL routing mechanism. A variety of RPL metrics were used in the previous studies to evaluate its performance in various network scenarios and it has been observed that several optimizations can be added to improve RPL functionalities.

While coordinating the forwarding of data packets, the responsibility of routing protocol is to create, manage, and maintain routing paths in a network [14]. The routing protocol is also responsible for transporting data packets successfully across the network from source to destination nodes. There are two types of routing protocols in WSN: Reactive and Proactive [14]. The reactive protocols are an event-driven protocol that provides routes by the trigger of an event or when needed.

Creating optimal routing paths and finding the shortest paths linking the source and destination nodes and nodes storing the paths on their routing table are some of the important tasks of any routing mechanism. The routing table size is usually measured in the number of routing entries in Kilobytes. RPL can work in both storing and non-storing mode and this enables very resource-constrained nodes (with little or no memory for routing table) to join an RPL network [15]. In non-storing mode, a node is not required to store the routing entries in its routing table and source routing is used to carry the routing information along the path. An RPL node stores the routes to all the routes downward in the tree and the size of the routing table can use more memory in large networks in the storing mode. However, these path entries depend on the OF in use because each uses different metrics to find the best path [16].

Control message overhead (CMO) [17] is very important in RPL as it is used to build, manage, and maintain the routing paths. The limited power nature of sensor node demands that optimal control messages are required and excess results in unnecessary power consumption leading to shortened operational network lifetime. The Trickle timer is used by RPL to regulate the generation of the control 
message to ensure optimal network performances [18]. When the network is unstable, more control messages are generated to build alternate routing paths and when the network is stable, the rate of control messages is reduced.

\section{Performance Evaluation}

The performance of RPL is evaluated based on its two Objective Functions; OF0 and MRHOF, and this will be performed with the RPL version of the Contiki OS; ContikiRPL. Several factors affect the performance of RPL such as the DIO minimum interval and doubling, duty cycling interval, and the frequency of application messages. These evaluations will be carried out with regards to the following metrics; Energy consumption, Network Latency, and Packet delivery ratio (PDR). The detail of these metrics is presented in section 3.1.

\subsection{Performance metrics}

The following metrics are used to evaluate the performance of RPL with regards to OF0 and MRHOF.

Packet Delivery Ratio (PDR): This is estimated as the number of packets received at the root node to the total number of packets sent by the source node. We take the average PDR of all the packets received successfully at the root node [19]. This is estimated by Eq. 1

$$
P D R=\frac{\text { Packets received }}{\text { Total packets sent }} \times 100 \%
$$

Power Consumption: This is the estimated total power consumption by a node for transmission, reception, low power/sleep mode, and data processing by the microcontroller [20]. See Eq. 2.

$$
P_{\text {Total }}=P_{T x}+P_{R x}+P_{M C U}+P_{\text {Listen }}
$$


Latency: The Latency is the time it takes for a packet to arrive at the root node from the source node [8]. It is estimated by Eq. 3.

$$
\text { Latency }=\text { Time packet arrive - Time packet sent }
$$

\subsection{Comparison of RPL Objective Functions}

RPL uses OFs (OF0 and MRHOF) to generates optimal routing paths to the root node using certain optimization criteria such as hop count, ETX, latency, residual energy, and link quality. By default, OF0 is assigned to use the $\mathrm{HC}$ metric, while MRHOF is assigned to use the ETX metrics to compute the optimal routing path. Node also uses the set OF metrics to select the next-hop parent node leading to the root node as well as node ranking. Contiki implements these two objective functions; OF0 selects the path to the root with minimum hops. This can be achieved by comparing the ranks of parents by using a 16-bit rank in units of 256 (min_hoprankinc) which allows a maximum of 255 hops [21]. On the other hand, The ETX metric used by MRHOF uses the expected number of transmissions required to successfully transmit a packet on the link. The path with the shortest ETX is selected as the preferred routing path to the root node.

\subsection{Simulation and Network Setup}

The simulation is performed on the Contiki OS. A total number of one hundred (100) nodes are scattered on a $100 \times 100$-meter square (m2) sensor field with one root node acting as the network coordinator [8], [22]. The number of nodes is varied incrementally from 10 nodes to 100 nodes as this enables us to estimate the behavior and performance of RPL with an increasing number of nodes. The Zolertia Z1 mote is used for the nodes [23], whose firmware is also embedded in Contiki. Z1 runs on the MSP430 microcontroller with the CC2420 radio transceiver which is compliant with the IEEE 802.15.4 standard [24]. The Unit Disk Graph Model (UDGM) with distance loss for the radio medium model is adopted. Table 1 shows the simulation configurations and settings. 
Table 1: Simulation Configurations and Settings

\begin{tabular}{|l|l|}
\hline Parameter & Value \\
\hline $\begin{array}{l}\text { Operating } \\
\text { System/Simulator }\end{array}$ & Contiki OS version3/Cooja \\
\hline Radio Medium Model & Unit Disk Graph Medium (UDGM): Distance Loss \\
\hline $\begin{array}{l}\text { Operating Carrier } \\
\text { Frequency }\end{array}$ & $2.4 \mathrm{GHz}$ \\
\hline Channel Data Rate & $250 \mathrm{kbps}$ \\
\hline Routing Protocols & SPPB-RPL, RPL, LOADng and LOADng-CTP \\
\hline Number of Nodes & 300 \\
\hline Tx/Rx Range & $200 \mathrm{~m}$ x 200m \\
\hline Simulation Time & $2000 \mathrm{sec}$ \\
\hline Packet Size & 127 bytes \\
\hline PHY and MAC Protocol & IEEE 802.15 .4 with CSMA/CA \\
\hline Mote Type & Z1 (Zolertia) \\
\hline
\end{tabular}

\section{Results and discussions}

This section discusses the results of the performance evaluation between OF0 and MRHOF.

\subsection{Packet Delivery Ratio - OFO vs MRHOF}

Fig. 3 shows the PDR performance of MRHOF and OF0. Both OFs show decreasing PDR with an increasing number of nodes. OF0 has its peak value of $100 \%$ at the 10 nodes and the least value of $71 \%$ at the 100 nodes. This decrease results from the increase in the collision domain with an increasing

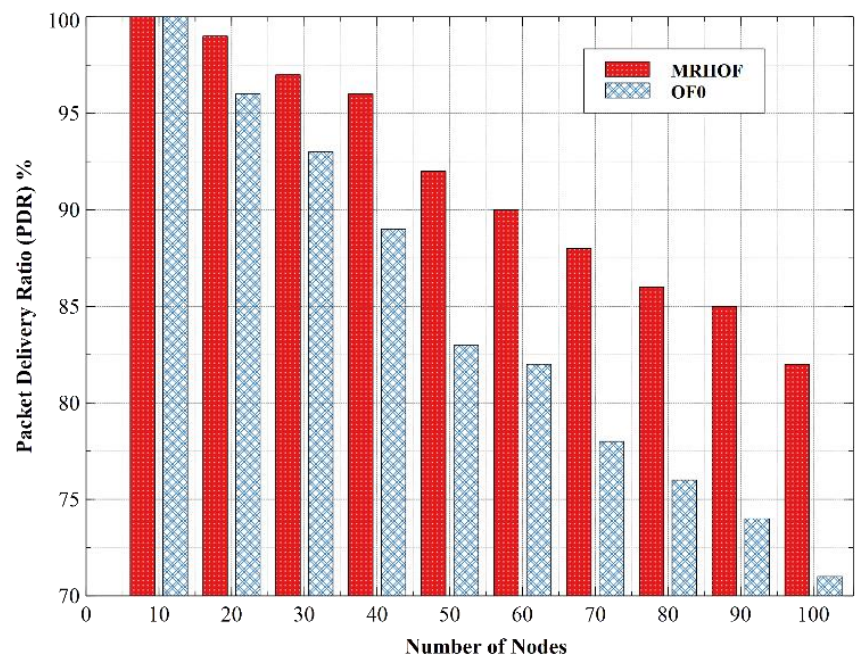

Fig. 3: Packet Delivery Ratio (PDR) 
number of pf nodes. Subsequently, the OF0 uses HC as a metric to estimate the shortest path to the root node without considerations for link quality. On the other hand, MRHOF presents a better PDR value with its highest value of $100 \%$ at the 10 nodes and $82 \%$ at the 100 nodes. Though MRHOF also experiences the effect of increasing the collision domain with increasing node numbers, its improved performance is attributed to the meticulous way it creates routing paths to the root node by considering link quality, and path cost. Data is routed through the least path cost which improves the performance of the network and encourages prolonged network lifetime.

\subsection{Power Consumption}

The power consumption of nodes for OF0 and MRHOF is shown in Fig. 4. Both OFs show increasing power consumption with an increasing number of nodes. OF0 has its least value of $0.412 \mathrm{~mW}$ at 10 nodes and $3.112 \mathrm{~mW}$ at 100 nodes. This increasing power value results from the increasing collision domain with an increasing number of nodes. This results in dropped packets and eventual retransmission of the packet. Moreover, the $\mathrm{HC}$ mechanism used by $\mathrm{OF} 0$ to compute the routing path is not optimal. MRHOF shows better power consumption values with the least consumption of $0.301 \mathrm{~mW}$ at 10 nodes and 2.801 at 100 nodes. The optimal power consumption is a result of the efficient routing mechanism of MRHOF which considers the quality of the link between adjacent nodes before selecting a preferred next-hop

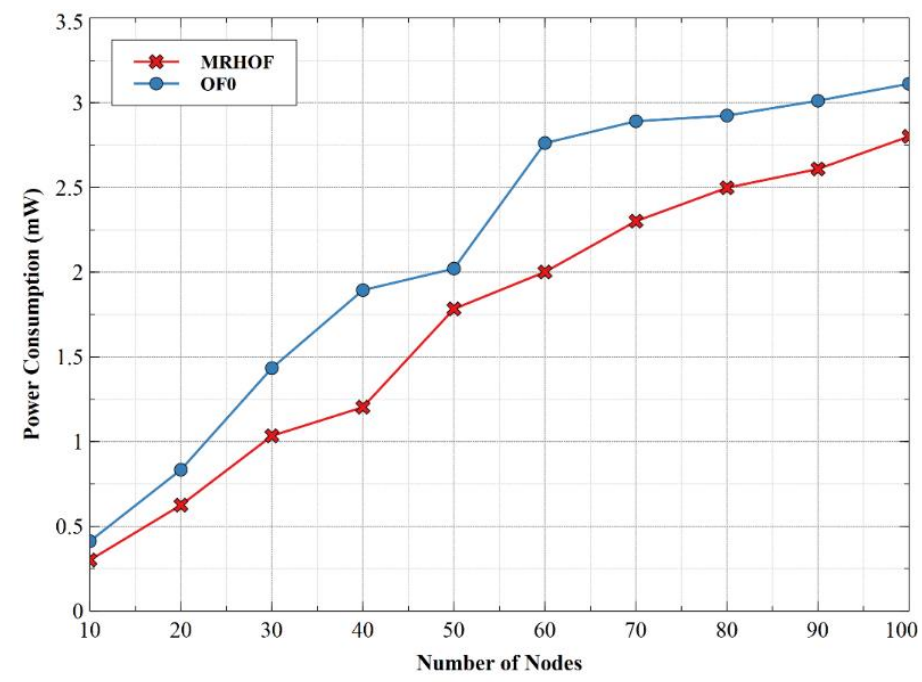

Fig. 4: Power Consumption 
node. This helps to improve network performances.

\subsection{Latency}

The latency readings are shown in Fig. 5. Both OFs shows increasing latency with an increasing number of nodes. OF0 presents its least value of $21 \mathrm{~ms}$ at 10 nodes and $102 \mathrm{~ms}$ at 100 nodes. This increasing endto-end delay (latency) is a result of increasing packet collision due to increasing node number. The suboptimal route computation mechanism of OF0 also contributes to increasing network latency. On the other hand, MRHOF presents better performance with the least value of $7 \mathrm{~ms}$ at 10 nodes and $85 \mathrm{~ms}$ at 100 nodes. The improved latency performance results from the optimal route generation mechanism which uses ETX to estimate the path with the least path cost. This results in overall optimal network performances.

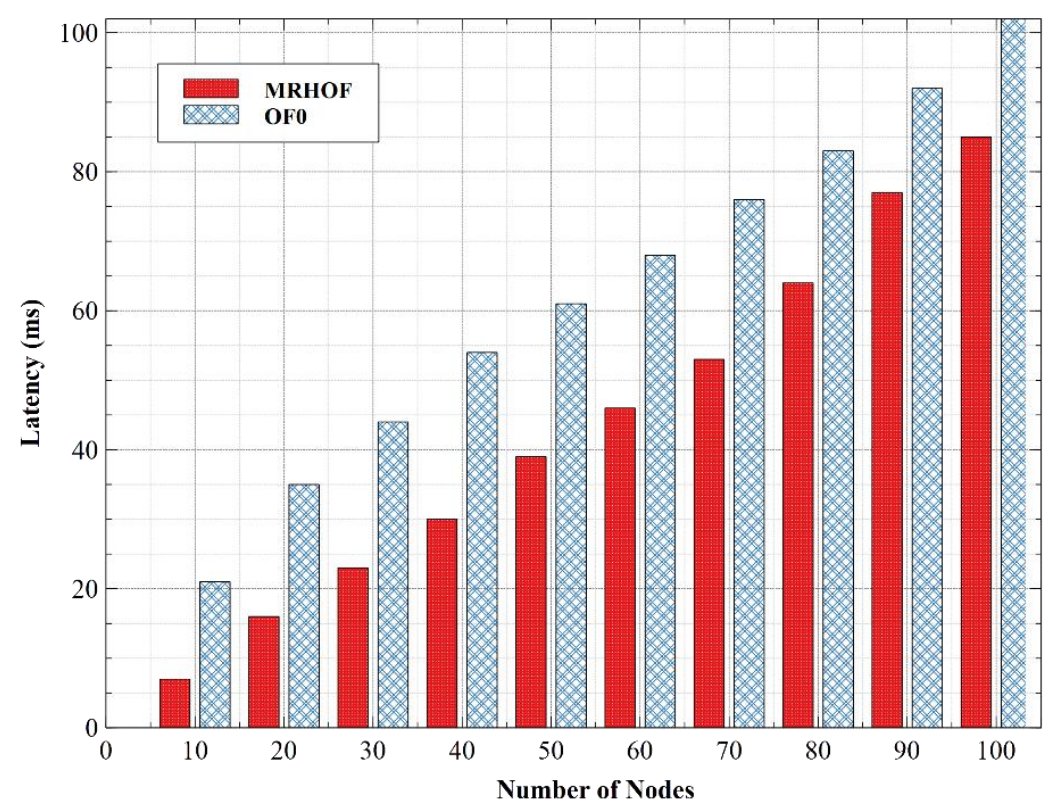

Fig. 5: Network Latency 


\section{Conclusion and future work}

In this research, we have presented the Routing Protocol for Low-power and Lossy Networks (RPL) for WSN. The study also presents the working mechanisms of RPL. Virtual routing graphs are generated by the ICMP control messages, which are also used to maintain the routing path. Due to the lossy nature of the wireless links, from time to time the paths are dynamically regenerated, to ensure optimal low cost and shortest path costs linking the source to the root nodes. Results obtained from the performance evaluation shows that MRHOF outperformed OF0 for PDR, power consumption, and latency. The improved performance of MRHOF results from the precise approach of building routing paths before engaging in data transmission. While OF uses HC, MRHOF uses ETX, which considers the least transmission cost for the selection of optimal path in the presence of other possible paths. From the results, it is clear that MRHOF outperformed OF0 in with regards to the considered metrics, hence considered as the best OF. For events that are time-sensitive with critical data, MRHOF can be used, and for events that are delay-tolerant, OF0 can be used. Our future work focus on how these Objective Functions can be used to transmit heterogeneous data for optimal routing experience.

\section{References}

[1] M. Frei, C. Deb, R. Stadler, Z. Nagy, and A. Schlueter, "Wireless sensor network for estimating building performance,” Autom. Constr., vol. 111, no. June 2019, p. 103043, Mar. 2020, doi: 10.1016/j.autcon.2019.103043.

[2] M. S. Bensaleh, R. Saida, Y. H. Kacem, and M. Abid, "Wireless Sensor Network Design Methodologies: A Survey,” J. Sensors, vol. 2020, 2020, doi: 10.1155/2020/9592836.

[3] H. Kharrufa, A. H. Kemp, and S. Member, "RPL-based routing protocols in IoT applications : A Review," 2019.

[4] H. Tall, G. Chalhoub, N. Hakem, and M. Misson, "Load balancing routing with queue overflow prediction for WSNs," Wirel. Networks, vol. 25, no. 1, pp. 229-239, 2019, doi: 10.1007/s11276- 
017-1554-6.

[5] I. U. Onwuegbuzie, S. Abd Razak, I. Fauzi Isnin, T. S. J. Darwish, and A. Al-dhaqm, "Optimized backoff scheme for prioritized data in wireless sensor networks: A class of service approach,” PLoS One, vol. 15, no. 8, p. e0237154, Aug. 2020, doi: 10.1371/journal.pone.0237154.

[6] M. Farooq and D. Pesch, "Reduced Overhead Routing in Short-Range Low-Power and Lossy Wireless Networks," Sensors, vol. 19, no. 5, pp. 1240-1259, Mar. 2019, doi: 10.3390/s19051240.

[7] M. O. Farooq, C. J. Sreenan, K. N. Brown, and T. Kunz, "RPL-Based Routing Protocols For Multi-Sink Wireless Sensor Networks,” pp. 452-459, 2020.

[8] S. S. Solapure and H. H. Kenchannavar, "Design and analysis of RPL objective functions using variant routing metrics for IoT applications," Wirel. Networks, vol. 26, no. 6, pp. 4637-4656, Aug. 2020, doi: 10.1007/s11276-020-02348-6.

[9] H. Al-Kashoash, Congestion Control for 6LoWPAN Wireless Sensor Networks: Toward the Internet of Things. Cham: Springer International Publishing, 2020.

[10] I. U. Onwuegbuzie, S. A. Razak, and I. F. Isnin, “Control Messages Overhead Impact on Destination Oriented Directed Acyclic Graph-A Wireless Sensor Networks Objective Functions Performance Comparison,” J. Comput. Theor. Nanosci., vol. 17, no. 2, pp. 1227-1235, Feb. 2020, doi: $10.1166 /$ jctn.2020.8794.

[11] A. Musaddiq, Y. Bin Zikria, Zulqarnain, and S. W. Kim, "Routing protocol for Low-Power and Lossy Networks for heterogeneous traffic network," EURASIP J. Wirel. Commun. Netw., vol. 2020, no. 1, pp. 21-44, Dec. 2020, doi: 10.1186/s13638-020-1645-4.

[12] T. Herrera and F. Nunez, "Design and Prototyping of a Thread Border Router Based on a Non Network-Co-Processor Architecture," IEEE Access, vol. 8, pp. 456-469, 2020, doi: 10.1109/ACCESS.2020.2983734.

[13] S. Junior, A. Riker, B. Silvestre, W. Moreira, A. Oliveira-Jr, and V. Borges, "DYNASTIDynamic multiple RPL instances for multiple IoT applications in smart city," Sensors 
(Switzerland), vol. 20, no. 11, pp. 1-23, 2020, doi: 10.3390/s20113130.

[14] J. V. V. Sobral, J. J. P. C. Rodrigues, R. A. L. Rabêlo, J. Al-Muhtadi, and V. Korotaev, "Routing Protocols for Low Power and Lossy Networks in Internet of Things Applications," Sensors, vol. 19, no. 9, pp. 2144-2184, May 2019, doi: 10.3390/s19092144.

[15] S.-W. Min, S.-H. Chung, H.-J. Lee, and Y.-V. Ha, "Downward traffic retransmission mechanism for improving reliability in RPL environment supporting mobility," Int. J. Distrib. Sens. Networks, vol. 16, no. 1, p. 155014772090360, Jan. 2020, doi: 10.1177/1550147720903605.

[16] F. T. Zuhra, K. B. A. Bakar, A. A. Arain, U. A. Khan, and A. R. Bhangwar, "MIQoS-RP: MultiConstraint Intra-BAN, QoS-Aware Routing Protocol for Wireless Body Sensor Networks," IEEE Access, vol. 8, pp. 1276-1285, 2020, doi: 10.1109/ACCESS.2020.2997402.

[17] J. V. V. Sobral, J. J. P. C. Rodrigues, R. A. L. Rabêlo, K. Saleem, and V. Furtado, "LOADngIoT: An Enhanced Routing Protocol for Internet of Things Applications over Low Power Networks," Sensors, vol. 19, no. 1, p. 150, Jan. 2019, doi: 10.3390/s19010150.

[18] H. Farag, P. Österberg, and M. Gidlund, "Congestion control and traffic differentiation for heterogeneous 6tisch networks in IIoT," Sensors (Switzerland), vol. 20, no. 12, pp. 1-25, 2020, doi: $10.3390 / \mathrm{s} 20123508$.

[19] M. Amirinasab Nasab, S. Shamshirband, A. Chronopoulos, A. Mosavi, and N. Nabipour, “Energy-Efficient Method for Wireless Sensor Networks Low-Power Radio Operation in Internet of Things," Electronics, vol. 9, no. 2, pp. 320-333, Feb. 2020, doi: 10.3390/electronics9020320.

[20] B. Zhang and D. J. Hu, "Research on the construction and simulation of PO-Dijkstra algorithm model in parallel network of multicore platform," Eurasip J. Wirel. Commun. Netw., vol. 2020, no. 1, pp. 85-99, Dec. 2020, doi: 10.1186/s13638-020-01680-х.

[21] H. Lamaazi, N. Benamar, and A. J. Jara, "Study of the impact of designed objective function on the RPL-based routing protocol," in Lecture Notes in Electrical Engineering, 2017, vol. 397, pp. 67-80, doi: 10.1007/978-981-10-1627-1_6.

[22] B. Al-Ghamdi, M. Ayaida, and H. Fouchal, "Performance Evaluation of Scheduling Approaches 
for Wireless Sensor Networks," Wirel. Pers. Commun., vol. 110, no. 3, pp. 1089-1108, Feb. 2020, doi: 10.1007/s11277-019-06775-3.

[23] Zolertia, “The Z1 mote · Zolertia/Resources Wiki · GitHub,” Jul. 18, 2018. https://github.com/Zolertia/Resources/wiki/The-Z1-mote (accessed Jul. 11, 2020).

[24] W. Badreddine, C. Chaudet, F. Petruzzi, and M. Potop-Butucaru, "Broadcast strategies and performance evaluation of IEEE 802.15.4 in wireless body area networks WBAN," Ad Hoc Networks, vol. 97, p. 102006, 2020, doi: 10.1016/j.adhoc.2019.102006. 